\title{
Remarks on the Regularity Criterion of the Navier-Stokes Equations with Nonlinear Damping
}

\author{
Weihua Wang ${ }^{1}$ and Guopeng Zhou ${ }^{2}$ \\ ${ }^{1}$ School of Mathematics and Statistics, Hubei University, Wuhan 430062, China \\ ${ }^{2}$ Institute of Engineering and Technology, Hubei University of Science and Technology, Xianning 437100, China \\ Correspondence should be addressed to Guopeng Zhou; zhgpeng@163.com
}

Received 16 March 2015; Accepted 26 May 2015

Academic Editor: Zenghui Wang

Copyright (c) $2015 \mathrm{~W}$. Wang and G. Zhou. This is an open access article distributed under the Creative Commons Attribution License, which permits unrestricted use, distribution, and reproduction in any medium, provided the original work is properly cited.

This paper is concerned with the regularity criterion of weak solutions to the three-dimensional Navier-Stokes equations with nonlinear damping in critical weak $L^{q}$ spaces. It is proved that if the weak solution satisfies $\int_{0}^{T}\left(\left(\left\|\nabla u_{1}\right\|_{L^{q, \infty}}^{2 q /(2 q-3)}+\left\|\nabla u_{2}\right\|_{L^{q, \infty}}^{2 q /(2 q-3)}\right) /(1+\right.$ $\left.\left.\ln \left(e+\|\nabla u\|_{L^{2}}^{2}\right)\right)\right) d s<\infty, q>3 / 2$, then the weak solution of Navier-Stokes equations with nonlinear damping is regular on $(0, T]$.

\section{Introduction}

In this study we consider the Cauchy problem of the threedimensional Navier-Stokes equations with the nonlinear damping

$$
\begin{aligned}
\partial_{t} u+(u \cdot \nabla) u+\nabla \pi+|u|^{r-2} u & =\Delta u, \\
\nabla \cdot u & =0,
\end{aligned}
$$

together with the initial data

$$
u(x, 0)=u_{0},
$$

where $u=\left(u_{1}(x, t), u_{2}(x, t), u_{3}(x, t)\right)$ and $\pi(x, t)$ denote the unknown velocity fields and the unknown pressure of the fluid. $|u|^{r-2} u, r>2$ is the nonlinear damping. Moreover,

$$
\nabla=\left(\partial_{1}, \partial_{2}, \partial_{3}\right)
$$

represents the gradient operator,

$$
\Delta=\nabla \cdot \nabla=\sum_{i=1}^{3} \frac{\partial^{2} u(x, t)}{\partial x_{i}^{2}}
$$

denotes the Laplacian operator, and

$$
\begin{aligned}
(u \cdot \nabla) u & =\sum_{i=1}^{3} u_{i} \frac{\partial u(x, t)}{\partial x_{i}}, \\
\nabla \cdot u & =\sum_{i=1}^{3} \frac{\partial u_{i}(x, t)}{\partial x_{i}} .
\end{aligned}
$$

The mathematical model (1) is from the resistance to the motion of the flows. It describes various physical situations such as drag or friction effects, porous media flow, and some dissipative mechanisms $[1,2]$. When the nonlinear damping term $|u|^{r-2} u$ in (1) disappears, the system reduces the classic Navier-Stokes equations $[3,4]$

$$
\begin{aligned}
\partial_{t} u+(u \cdot \nabla) u+\nabla \pi & =\Delta u, \\
\nabla \cdot u & =0 .
\end{aligned}
$$

In the mathematical viewpoint, therefore, Navier-Stokes equations with the nonlinear damping are a modification of the classic Navier-Stokes equations. There is a large literature on the well-posedness and large time behavior for solutions of Navier-Stokes equations with the nonlinear damping (see $[1,5,6])$.

However it is not known whether the weak solution of Navier-Stokes equations with the nonlinear damping (1) is 
regular or smooth for a given smooth and compactly supported initial velocity $u_{0}$. Fortunately, the regularity of weak solutions for Navier-Stokes equations with the nonlinear damping (1) can be derived when certain growth conditions are satisfied. This is known as a regularity criterion problem. Recently, Zhou [7] studied the regularity criterion for weak solutions for Navier-Stokes equations with the nonlinear damping (1) in critical Lebesgue spaces. That is, if a weak solution $u$ of Navier-Stokes equations with the nonlinear damping (1) satisfies

$$
\nabla u \in L^{p}\left(0, T ; L^{q}\left(\mathbb{R}^{3}\right)\right) \quad \text { for } \frac{2}{p}+\frac{3}{q}=2,3<p \leq \infty,
$$

then the weak solution is smooth on $(0, T]$.

Since Navier-Stokes equations with the nonlinear damping (1) are a modification of the classic Navier-Stokes equations, it is necessary to mention some regularity criteria of weak solutions for Navier-Stokes equations and related fluid models $[8,9]$. As for this direction, the first result of NavierStokes equations is studied by $\mathrm{He}[10]$ and improved by Dong and Zhang [11], Pokorý [12, 13], and Zhou [14]. One may also refer to some interesting regularity criteria on related fluid models (see [15] and the references therein).

The main purpose of this paper is to investigate the regularity criteria of weak solutions with the aid of two components of velocity fields in critical weak $L^{q}$ space. To do so, we recall the definition of the weak solution of NavierStokes equations with the nonlinear damping (1).

Definition 1. A measurable function $u(x, t)$ is called a weak solution of Navier-Stokes equations with the nonlinear damping (1) on $(0, T)$ if $u$ satisfies the following properties:

(i) $u \in L^{\infty}\left(0, T ; L^{2}\left(\mathbb{R}^{3}\right)\right)$ and $\nabla u \in L^{2}\left(0, T ; L^{2}\left(\mathbb{R}^{3}\right)\right)$;

(ii) for any $\phi \in C_{0}^{\infty}\left(\mathbb{R}^{3} \times[0, T)\right)$ with $\nabla \cdot \phi=0$

$$
\begin{aligned}
& \int_{0}^{T} \int_{\mathbb{R}^{3}}\left(u \cdot \partial_{t} \phi-\nabla u \cdot \nabla \phi+\nabla \phi: u \otimes u+|u|^{r-2} u \phi\right) d x d t \\
& \quad=-\int_{\mathbb{R}^{3}} u_{0} \phi(0) d x ;
\end{aligned}
$$

(iii) $\nabla \cdot u=0$ in the distribution space $\mathscr{D}^{\prime}\left((0, T) \times \mathbb{R}^{3}\right)$;

(iv) $u$ satisfies the energy inequality for $0 \leq t \leq T$

$$
\begin{aligned}
& \|u(t)\|_{L^{2}}^{2}+2 \int_{0}^{t} \int_{\mathbb{R}^{3}}|\nabla u(x, s)|^{2} d x d s \\
& \quad+2 \int_{0}^{t} \int_{\mathbb{R}^{3}}|u(x, s)|^{p} d x d s \leq\left\|u_{0}\right\|_{L^{2}}^{2} .
\end{aligned}
$$

The main result on the regularity criteria of the weak solutions of Navier-Stokes equations with the nonlinear damping (1) is now read.

Theorem 2. Suppose $u_{0} \in L^{2}\left(\mathbb{R}^{3}\right) \cap \dot{H}^{1}\left(\mathbb{R}^{3}\right)$ and $u$ is a weak solution of Navier-Stokes equations with the nonlinear damping (1) in (0,T). If any two components of velocity fields satisfy

$$
\int_{0}^{T} \frac{\left\|\nabla u_{1}\right\|_{L^{q, \infty}}^{2 q /(2 q-3)}+\left\|\nabla u_{2}\right\|_{L^{q, \infty}}^{2 q /(2 q-3)}}{1+\ln \left(e+\|\nabla u\|_{L^{2}}^{2}\right)} d s<\infty, \quad q>\frac{3}{2},
$$

then $u$ is smooth on $(0, T]$.

This result improves the earlier regularity criterion involving (7). Furthermore, Theorem 2 also implies the following regularity criterion for weak solutions of NavierStokes equations with the nonlinear damping (1).

Theorem 3. Suppose $u_{0} \in L^{2}\left(\mathbb{R}^{3}\right) \cap \dot{H}^{1}\left(\mathbb{R}^{3}\right)$ and $u$ is a weak solution of Navier-Stokes equations with the nonlinear damping (1) in $(0, T)$. If any two components of velocity fields satisfy

$$
\int_{0}^{T}\left\|\nabla u_{1}\right\|_{L^{q, \infty}}^{2 q /(2 q-3)}+\left\|\nabla u_{2}\right\|_{L^{q, \infty}}^{2 q /(2 q-3)} d s<\infty, \quad q>\frac{3}{2},
$$

then $u$ is smooth on $(0, T]$.

Remark 4. The main idea in the proof of Theorem 2 is borrowing from the argument of previous results on classic Navier-Stokes equations [16] and together with energy methods.

\section{Preliminaries}

To start with, let us recall the definitions of some functional spaces. $L^{q}\left(\mathbb{R}^{3}\right)$ with $1 \leq q \leq \infty$ is a Lebesgue space under the norm

$$
\|g\|_{L^{q}}= \begin{cases}\left(\int_{\mathbb{R}^{3}}|g(x)|^{q} d x\right)^{1 / q}, & 1 \leq q<\infty, \\ \operatorname{ess} \sup _{x \in \mathbb{R}^{3}}|g(x)|, & q=\infty,\end{cases}
$$

and $\dot{H}^{m}\left(\mathbb{R}^{3}\right)$ the Hilbert space

$$
\left\{g \in L^{2}\left(\mathbb{R}^{3}\right) ;\left\|\nabla^{m} g\right\|_{L^{2}}<\infty\right\} .
$$

To define the Lorenz space $L^{p, q}\left(\mathbb{R}^{3}\right)$ with $1 \leq p, q \leq \infty$, $g \in L^{p, q}\left(\mathbb{R}^{3}\right)$ if and only if

$$
\begin{aligned}
& \|g\|_{L^{p, q}}=\left(\int_{0}^{\infty} t^{q}(m(g, t))^{q / p} \frac{d t}{t}\right)^{1 / q}<\infty \\
& \| \text { for } 1 \leq q<\infty, \\
& \|g\|_{L^{p, \infty}}=\sup _{t \geq 0}\left(t(m(g, t))^{1 / p}\right)<\infty \quad \text { for } q=\infty,
\end{aligned}
$$

where

$$
m(f, t):=m\left\{x \in \mathbb{R}^{3}:|g(x)|>t\right\}
$$

is Lebesgue measure of the set $\left\{x \in \mathbb{R}^{3}:|g(x)|>t\right\}$. 
Actually Lorentz space $L^{p, q}\left(R^{3}\right)$ may be alternatively defined by real interpolation (see Triebel [17])

$$
L^{p, q}\left(\mathbb{R}^{3}\right)=\left(L^{p_{1}}\left(\mathbb{R}^{3}\right), L^{p_{2}}\left(\mathbb{R}^{3}\right)\right)_{\theta, q}
$$

with

$$
\begin{aligned}
\frac{1}{p} & =\frac{1-\theta}{p_{1}}+\frac{\theta}{p_{2}}, \quad 1 \leq p_{1}<p<p_{2} \leq \infty \\
0 & <\theta<1 .
\end{aligned}
$$

In particular, $\|g\|_{L^{q, \infty}}$ is equivalently to the norm

$$
\sup _{0<|S|<\infty}|S|^{1 / q-1} \int_{S}|g(x)| d x .
$$

Furthermore, the definition implies the continuous relationship

$$
L^{q}\left(\mathbb{R}^{3}\right) \hookrightarrow L^{q, \infty}\left(\mathbb{R}^{3}\right), \quad 1<q<\infty .
$$

In fact it is easy to check and thus it is readily seen that

$$
\begin{gathered}
|x|^{-3 / q} \bar{\epsilon} L^{q}\left(\mathbb{R}^{3}\right), \\
\text { but }|x|^{-3 / q} \in L^{q, \infty}\left(\mathbb{R}^{3}\right) .
\end{gathered}
$$

We also recall the Hölder inequality in Lorentz space which plays an important role in the next section.

Lemma 5 (O'Neil [18]). Let $f \in L^{p_{1}, q_{1}}\left(\mathbb{R}^{3}\right)$ and $g \in L^{p_{2}, q_{2}}\left(\mathbb{R}^{3}\right)$ with

$$
\begin{array}{ll}
1 \leq p_{1}, & p_{2} \leq \infty, \\
1 \leq q_{1}, & q_{2} \leq \infty .
\end{array}
$$

Then $f g \in L^{p, q}\left(\mathbb{R}^{3}\right)$ satisfies the Hölder inequality of Lorentz spaces

$$
\|f g\|_{L^{p, q}} \leq C\|f\|_{L^{p_{1}, q_{1}}}\|g\|_{L^{p_{2}, q_{2}}}
$$

where

$$
\begin{aligned}
& \frac{1}{p}=\frac{1}{p_{1}}+\frac{1}{p_{2}} \\
& \frac{1}{q} \leq \frac{1}{q_{1}}+\frac{1}{q_{2}}
\end{aligned}
$$

\section{A Priori Estimates}

In this section we will prove a priori estimates for smooth solutions of (1) described in the following.

Theorem 6. Let $T>0$, let $u_{0} \in L^{2}\left(\mathbb{R}^{3}\right) \cap \dot{H}^{1}\left(\mathbb{R}^{3}\right)$, and let $u$ be a local smooth solution of the Navier-Stokes equations with the nonlinear damping (1). If u also satisfies (11), namely,

$$
\int_{0}^{T} \frac{\left\|\nabla u_{1}\right\|_{L^{q, \infty}}^{2 q /(2 q-3)}+\left\|\nabla u_{2}\right\|_{L^{q, \infty}}^{2 q /(2 q-3)}}{1+\ln \left(e+\|\nabla u\|_{L^{2}}^{2}\right)} d s<\infty, \quad q>\frac{3}{2},
$$

then the a priori estimate

$$
\sup _{0<t<T}\|u(t)\|_{L^{4}} \leq C
$$

holds true.

Proof of Theorem 6. Multiplying both sides of the NavierStokes equations with the nonlinear damping (1) with $\Delta u$ and integrating in $\mathbb{R}^{3}$, we have

$$
\begin{aligned}
& \frac{1}{2} \frac{d}{d t} \int_{\mathbb{R}^{3}}|\nabla u|^{2} d x+\int_{\mathbb{R}^{3}}|\Delta u|^{2} d x \\
& \quad+(r-1) \int_{\mathbb{R}^{3}}|u|^{r-2}|\nabla u|^{2} d x \\
& =-\int_{\mathbb{R}^{3}} u \cdot \nabla u \Delta u d x,
\end{aligned}
$$

where we have used

$$
\int_{\mathbb{R}^{3}} \nabla p \Delta u d x=-\int_{\mathbb{R}^{3}} p \Delta(\operatorname{div} u) d x=0 .
$$

For the right hand side of (26) we have

$$
\begin{aligned}
-\int_{\mathbb{R}^{3}} u \cdot \nabla u \Delta u d x= & -\sum_{i, j, k=1}^{3} \int_{\mathbb{R}^{3}} u_{i} \partial_{i} u_{j} \partial_{k k} u_{j} d x \\
= & \sum_{i, j, k=1}^{3} \int_{\mathbb{R}^{3}} \partial_{k}\left(u_{i} \partial_{i} u_{j}\right) \partial_{k} u_{j} d x \\
= & \sum_{i, j, k=1}^{3} \int_{\mathbb{R}^{3}} \partial_{k} u_{i} \partial_{i} u_{j} \partial_{k} u_{j} d x \\
& +\frac{1}{2} \sum_{i, j, k=1}^{3} \int_{\mathbb{R}^{3}} u_{i} \partial_{i}\left(\partial_{k} u_{j} \partial_{k} u_{j}\right) d x \\
= & \sum_{i, j, k=1}^{3} \int_{\mathbb{R}^{3}} \partial_{k} u_{i} \partial_{i} u_{j} \partial_{k} u_{j} d x \\
= & \sum_{i=1}^{2} \sum_{j, k=1}^{3} \int_{\mathbb{R}^{3}} \partial_{k} u_{i} \partial_{i} u_{j} \partial_{k} u_{j} d x \\
& +\sum_{k=1}^{2} \int_{\mathbb{R}^{3}}^{3} \partial_{k=1}^{3} \sum_{k=1} \int_{\mathbb{R}^{3}} \partial_{k} u_{3} \partial_{3} \partial_{3} u_{3} \partial_{k} \partial_{k} u_{3} d x \\
& +u_{j} d x \\
& \\
& \\
& \\
&
\end{aligned}
$$

where we have used the fact that the divergence-free condition

$$
\sum_{k=1}^{3} \partial_{k} u_{k}=0
$$


For $I_{1}$, we have

$$
\begin{aligned}
\left|I_{1}\right| & \leq\left|\sum_{i=1}^{2} \sum_{j, k=1}^{3} \int_{\mathbb{R}^{3}} \partial_{k} u_{i} \partial_{i} u_{j} \partial_{k} u_{j} d x\right| \\
& \leq \int_{\mathbb{R}^{3}}\left(\left|\nabla u_{1}\right|+\left|\nabla u_{2}\right|\right)|\nabla u|^{2} d x .
\end{aligned}
$$

For $I_{2}$, similarly we obtain

$$
\begin{aligned}
\left|I_{2}\right| & \leq\left|\sum_{j=1}^{2} \sum_{k=1}^{3} \int_{\mathbb{R}^{3}} \partial_{k} u_{3} \partial_{3} u_{j} \partial_{k} u_{j} d x\right| \\
& \leq \int_{\mathbb{R}^{3}}\left(\left|\nabla u_{1}\right|+\left|\nabla u_{2}\right|\right)|\nabla u|^{2} d x .
\end{aligned}
$$

$$
\begin{aligned}
\partial_{3} u_{3} & =-\partial_{2} u_{2}-\partial_{1} u_{1}, \\
\left|I_{3}\right| & \leq\left|\sum_{k=1}^{3} \int_{\mathbb{R}^{3}} \partial_{k} u_{3} \partial_{3} u_{3} \partial_{k} u_{3} d x\right| \\
& \leq\left|\sum_{k=1}^{3} \int_{\mathbb{R}^{3}} \partial_{k} u_{3}\left(-\partial_{2} u_{2}-\partial_{1} u_{1}\right) \partial_{k} u_{3} d x\right| \\
& \leq \int_{\mathbb{R}^{3}}\left(\left|\nabla u_{1}\right|+\left|\nabla u_{2}\right|\right)|\nabla u|^{2} d x .
\end{aligned}
$$

Plugging the estimates $I_{i}$ into the right hand side of (26), it follows that

$$
\begin{aligned}
& \frac{1}{2} \frac{d}{d t} \int_{\mathbb{R}^{3}}|\nabla u|^{2} d x+\int_{\mathbb{R}^{3}}|\Delta u|^{2} d x \\
& \quad+(r-1) \int_{\mathbb{R}^{3}}|u|^{r-2}|\nabla u|^{2} d x \\
& \leq C \int_{\mathbb{R}^{3}}\left(\left|\nabla u_{1}\right|+\left|\nabla u_{2}\right|\right)|\nabla u||\nabla u| d x .
\end{aligned}
$$

$$
\int_{\mathbb{R}^{3}}|\nabla u|^{2} d x \leq \int_{\mathbb{R}^{3}}\left|\nabla u_{0}\right|^{2} d x \exp \left\{\int_{0}^{T}\left(\frac{\left\|\nabla u_{1}\right\|_{L^{q, \infty}}^{2 q /(2 q-3)}+\left\|\nabla u_{2}\right\|_{L^{q, \infty}}^{2 q /(2 q-3)}}{1+\ln \left(e+\|\nabla u\|_{L^{2}}^{2}\right)}\left\{1+\ln \left(e+\|\nabla u\|_{L^{2}}^{2}\right)\right\}\right) d t\right\}
$$

Applying Hölder inequality and Young inequality, we have for the right hand side of (33)

$$
\begin{aligned}
& \int_{\mathbb{R}^{3}}\left(\left|\nabla u_{1}\right|+\left|\nabla u_{2}\right|\right)|\nabla u||\nabla u| d x \\
& \quad \leq C\left(\left\|\nabla u_{1}\right\|_{L^{q, \infty}}+\left\|\nabla u_{2}\right\|_{L^{q, \infty}}\right)\|\nabla u\|_{L^{2 q /(q-1), 2}}^{2} .
\end{aligned}
$$

Applying the Gagliardo-Nirenberg inequality in Lorentz spaces, that is,

$$
\|\nabla u\|_{L^{2 q /(q-1), 2}} \leq C\|\nabla u\|_{L^{2}}^{(2 q-3) / 2 q}\|\Delta u\|_{L^{2}}^{3 / 2 q},
$$

thus we have from (33)

$$
\begin{aligned}
& \frac{1}{2} \frac{d}{d t} \int_{\mathbb{R}^{3}}|\nabla u|^{2} d x+\int_{\mathbb{R}^{3}}|\Delta u|^{2} d x \\
& \quad+(r-1) \int_{\mathbb{R}^{3}}|u|^{r-2}|\nabla u|^{2} d x \\
& \quad \leq C\left(\left\|\nabla u_{1}\right\|_{L^{q, \infty}}+\left\|\nabla u_{2}\right\|_{L^{q, \infty}}\right)\|\nabla u\|_{L^{2}}^{(2 q-3) / q}\|\Delta u\|_{L^{2}}^{3 / q} \\
& \quad \leq \frac{1}{2}\|\Delta u\|_{L^{2}}^{2} \\
& \quad+C\left(\left\|\nabla u_{1}\right\|_{L^{q, \infty}}^{2 q /(2 q-3)}+\left\|\nabla u_{2}\right\|_{L^{q, \infty}}^{2 q /(2 q-3)}\right)\|\nabla u\|_{L^{2}}^{2}
\end{aligned}
$$

which implies

$$
\begin{aligned}
& \frac{d}{d t} \int_{\mathbb{R}^{3}}|\nabla u|^{2} d x+\int_{\mathbb{R}^{3}}|\Delta u|^{2} d x \\
& \quad \leq C\left(\left\|\nabla u_{1}\right\|_{L^{q, \infty}}^{2 q /(2 q-3)}+\left\|\nabla u_{2}\right\|_{L^{q, \infty}}^{2 q /(2 q-3)}\right)\|\nabla u\|_{L^{2}}^{2} .
\end{aligned}
$$

In particular,

$$
\begin{aligned}
& \frac{d}{d t} \int_{\mathbb{R}^{3}}|\nabla u|^{2} d x+\int_{\mathbb{R}^{3}}|\Delta u|^{2} d x \\
& \quad \leq C\left(\frac{\left\|\nabla u_{1}\right\|_{L^{q, \infty}}^{2 q /(2 q-3)}+\left\|\nabla u_{2}\right\|_{L^{q, \infty}}^{2 q /(2 q-3)}}{1+\ln \left(e+\|\nabla u\|_{L^{2}}^{2}\right)}\right) \\
& \quad \cdot\left(1+\ln \left(e+\|\nabla u\|_{L^{2}}^{2}\right)\right)\|\nabla u\|_{L^{2}}^{2} .
\end{aligned}
$$

Employing the Gronwall inequality, it follows that
Hence we have

$$
\begin{aligned}
& \ln \left(e+\int_{\mathbb{R}^{3}}|\nabla u|^{2} d x\right) \leq \ln \left(e+\int_{\mathbb{R}^{3}}\left|\nabla u_{0}\right|^{2} d x\right) \\
& +\int_{0}^{T}\left(\frac{\left\|\nabla u_{1}\right\|_{L^{q, \infty}}^{2 q /(2 q-3)}+\left\|\nabla u_{2}\right\|_{L^{q, \infty}}^{2 q /(2 q-3)}}{1+\ln \left(e+\|\nabla u\|_{L^{2}}^{2}\right)}\{1\right. \\
& \left.\left.\quad+\ln \left(e+\|\nabla u\|_{L^{2}}^{2}\right)\right\}\right) d t .
\end{aligned}
$$


Hence we obtain a priori estimates of $\nabla u$ :

$$
\text { ess } \sup _{0<t<T} \int_{\mathbb{R}^{3}}|\nabla u|^{2} d x<C\left(u_{0}\right) \text {. }
$$

\section{Proof of Theorem 2}

Under the a priori estimates in Theorem 6, we now are in a position to complete the proof of Theorem 2. Since $u_{0} \in$ $L^{2}\left(R^{3}\right) \cap H^{1}\left(\mathbb{R}^{3}\right)$ with $\nabla \cdot u_{0}=0$, by the existence theorem of local strong solutions to the Navier-Stokes equations with nonlinear damping $r>2$, there exist a constant $T^{*}>0$ and a unique smooth solution $\widetilde{u}$ of (1) satisfying (refer to [19])

$$
\tilde{u} \in B C\left(\left[0, T^{*}\right) ; H^{1}\right), \quad \tilde{u}(x, 0)=u_{0} .
$$

Note that the weak solution satisfies the energy inequality (9). It follows from the weak-strong uniqueness criterion that

$$
\tilde{u} \equiv u \text { on }\left[0, T^{*}\right) \text {. }
$$

Thus it is sufficient to show that $T^{*}=T$. Suppose that $T^{*}<$ $T$. Without loss of generality, we may assume that $T^{*}$ is the maximal existence time for $\widetilde{u}$. Since $\widetilde{u} \equiv u$ on $\left[0, T^{*}\right)$, by the assumptions (11),

$$
\int_{0}^{T} \frac{\left\|\nabla u_{1}\right\|_{L^{q, \infty}}^{2 q /(2 q-3)}+\left\|\nabla u_{2}\right\|_{L^{q, \infty}}^{2 q /(2 q-3)}}{1+\ln \left(e+\|\nabla u\|_{L^{2}}^{2}\right)} d s<\infty, \quad q>\frac{3}{2} .
$$

Therefore it follows from (25) that the existence time of $\tilde{u}$ can be extended after $t=T^{*}$ which contradicts the maximality of $t=T^{*}$.

This completes the proof of Theorem 2 .

\section{Conflict of Interests}

The authors declare that there is no conflict of interests regarding the publication of this paper.

\section{Acknowledgments}

This work is supported by the National Natural Science Foundation of China (Grant no. 61340042), the Natural Science Foundation of Hubei Province (Grant no. 2013CFC011), and the Project of the Education Department of Hubei Province (Grant nos. T201009 and D20132801).

\section{References}

[1] D. Bresch and B. Desjardins, "Existence of global weak solutions for a 2D viscous shallow water equations and convergence to the quasi-geostrophic model," Communications in Mathematical Physics, vol. 238, no. 1-2, pp. 211-223, 2003.

[2] Z.-Q. Luo, "Optimal convergence rates for solutions of the monopolar non-Newtonian flows," Abstract and Applied Analysis, vol. 2014, Article ID 738729, 6 pages, 2014.

[3] O. A. Ladyzhenskaya, The Mathematical Theory of Viscous Incompressible Fluids, Gorden Brech, New York, NY, USA, 1969.
[4] P. G. Lemarié-Rieusset, Recent Developments in the NavierStokes Problem, Chapman \& Hall/CRC, Boca Raton, Fla, USA, 2002.

[5] X. Cai and Q. Jiu, "Weak and strong solutions for the incompressible Navier-Stokes equations with damping," Journal of Mathematical Analysis and Applications, vol. 343, no. 2, pp. 799809, 2008.

[6] Y. Jia, X. Zhang, and B.-Q. Dong, "The asymptotic behavior of solutions to three-dimensional Navier-Stokes equations with nonlinear damping," Nonlinear Analysis. Real World Applications, vol. 12, no. 3, pp. 1736-1747, 2011.

[7] Y. Zhou, "Regularity and uniqueness for the 3D incompressible Navier-Stokes equations with damping," Applied Mathematics Letters, vol. 25, no. 11, pp. 1822-1825, 2012.

[8] J. Serrin, "On the interior regularity of weak solutions of the Navier-Stokes equations," Archive for Rational Mechanics and Analysis, vol. 9, pp. 187-195, 1962.

[9] I. Kukavica and M. Ziane, "One component regularity for the Navier-Stokes equations," Nonlinearity, vol. 19, no. 2, pp. 453469, 2006.

[10] C. He, "Regularity for solutions to the Navier-Stokes equations with one velocity component regular," Electronic Journal of Differential Equations, no. 29, 13 pages, 2002.

[11] B.-Q. Dong and Z. Zhang, "The BKM criterion for the 3D Navier-Stokes equations via two velocity components," Nonlinear Analysis: Real World Applications, vol. 11, no. 4, pp. 24152421, 2010.

[12] P. Penel and M. Pokorný, "Some new regularity criteria for the Navier-Stokes equations containing gradient of the velocity," Applications of Mathematics, vol. 49, no. 5, pp. 483-493, 2004.

[13] M. Pokorný, "On the result of He concerning the smoothness of solutions to the Navier-Stokes equations," Electronic Journal of Differential Equations, vol. 10, pp. 1-8, 2003.

[14] Y. Zhou, "A new regularity criterion for weak solutions to the Navier-Stokes equations," Journal de Mathématiques Pures et Appliquées, vol. 84, no. 11, pp. 1496-1514, 2005.

[15] B.-Q. Dong and Z.-M. Chen, "Regularity criteria of weak solutions to the three-dimensional micropolar flows," Journal of Mathematical Physics, vol. 50, no. 10, Article ID 103525, 13 pages, 2009.

[16] B.-Q. Dong and Z.-M. Chen, "Regularity criterion for weak solutions to the 3D Navier-Stokes equations via two velocity components," Journal of Mathematical Analysis and Applications, vol. 338, no. 1, pp. 1-10, 2008.

[17] H. Triebel, Interpolation Theory, Function Spaces, Differential Operators, vol. 18, North-Holland, Amsterdam, The Netherlands, 1978.

[18] R. O’Neil, "Convolution operators and $L(p, q)$ spaces," Duke Mathematical Journal, vol. 30, pp. 129-142, 1963.

[19] Y. Giga, "Solutions for semilinear parabolic equations in $L^{p}$ and regularity of weak solutions of the Navier-Stokes system," Journal of Differential Equations, vol. 62, no. 2, pp. 186-212, 1986. 


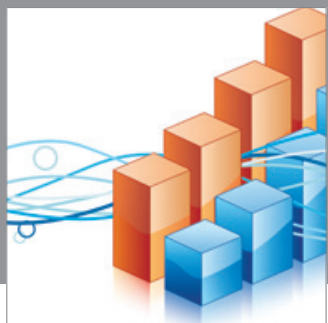

Advances in

Operations Research

mansans

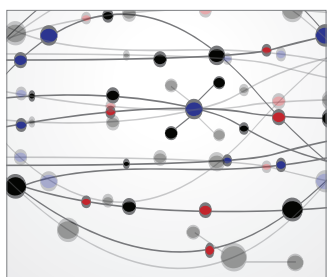

The Scientific World Journal
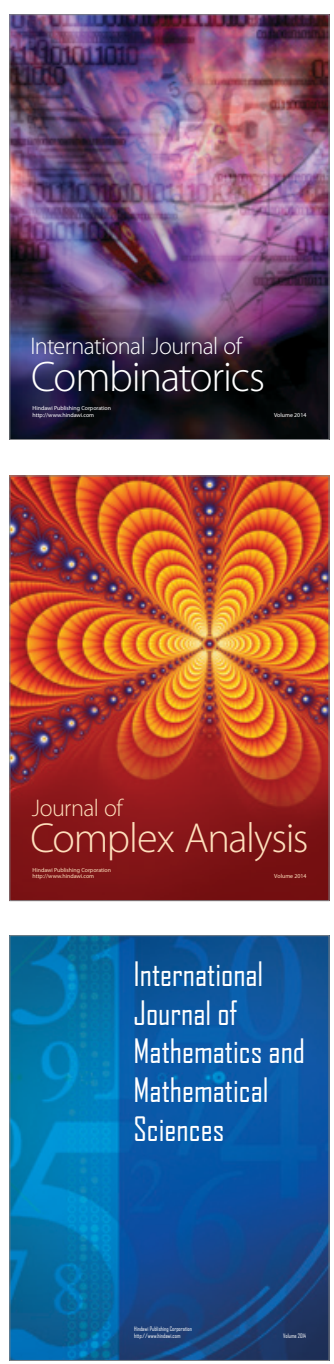
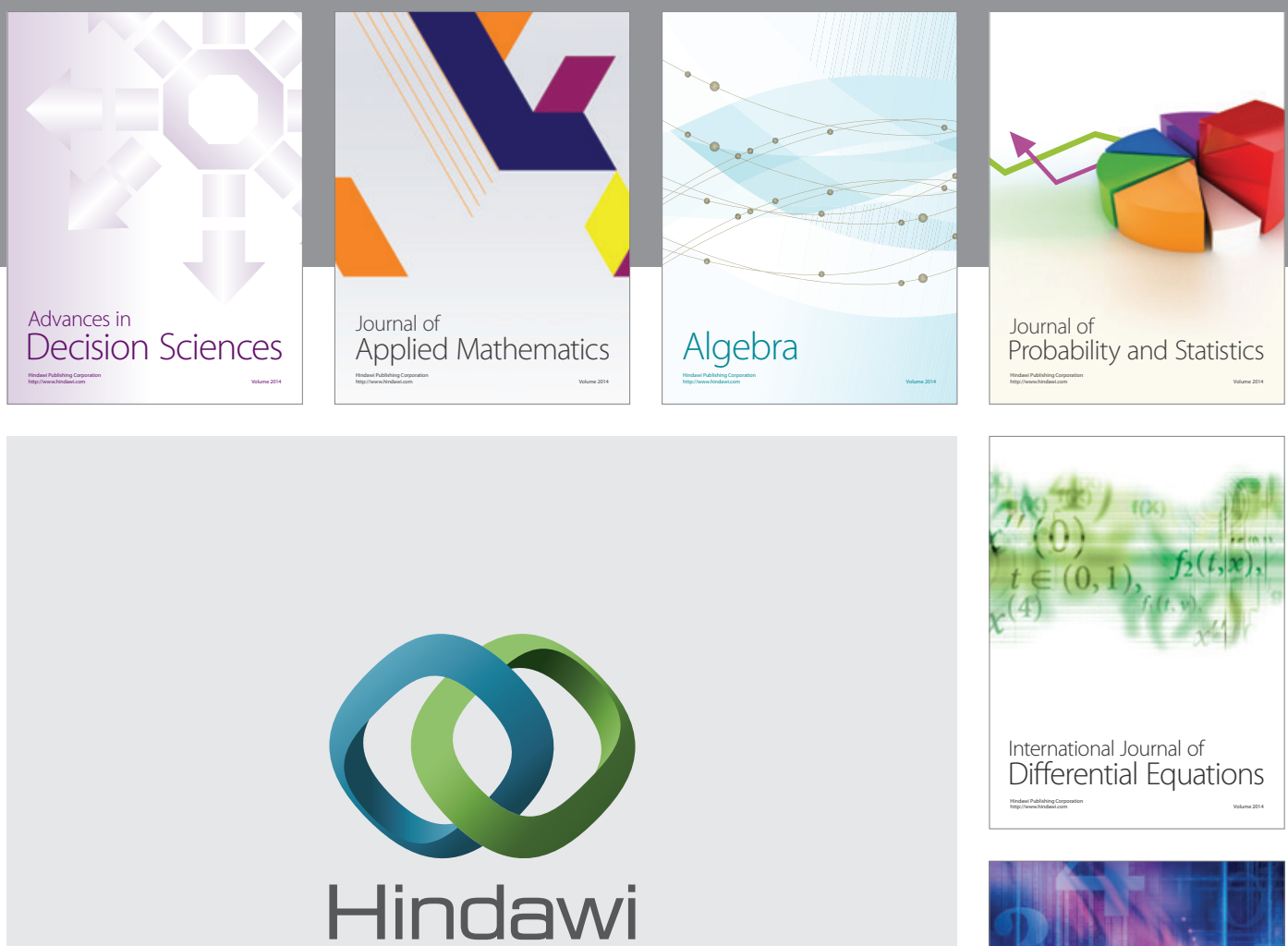

Submit your manuscripts at http://www.hindawi.com
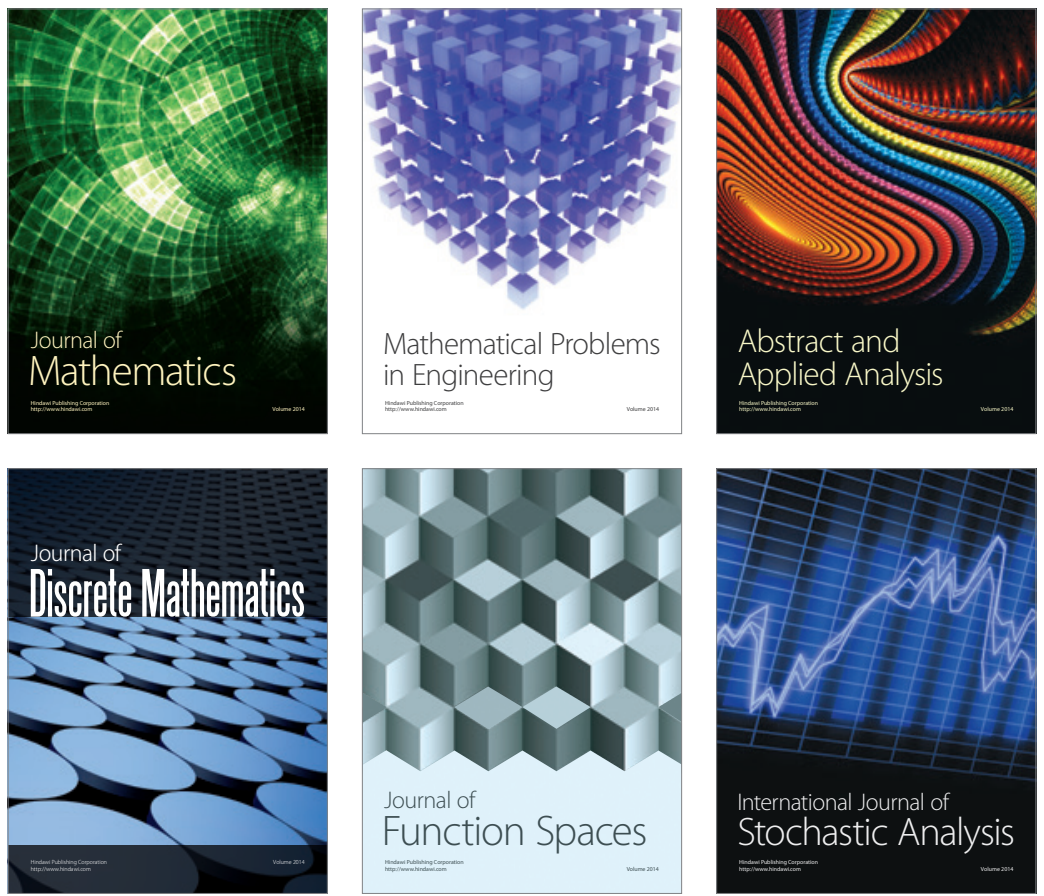

Journal of

Function Spaces

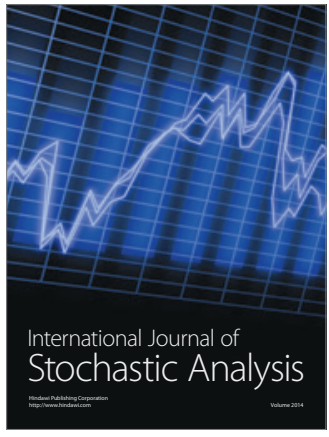

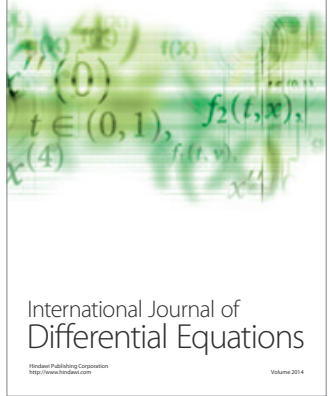
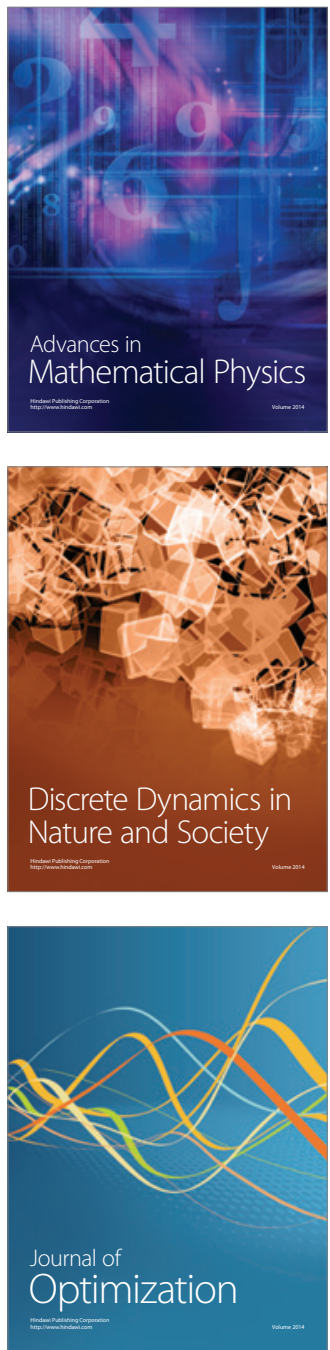